\title{
MASSES FROM AN INHOMOGENEOUS PARTIAL DIFFERENCE EQUATION WITH HIGHER-ORDER ISOSPIN CONTRIBUTIONS*
}

\author{
P. J. MASSON and J. JÄNECKE \\ Department of Physics, University of Michigan \\ Ann Arbor, Michigan 48109
}

\begin{abstract}
In the present work, a mass equation obtained as the solution of an inhomogeneous partial difference equation is used to predict masses of unknown neutron-rich and proton-rich nuclei. The inhomogeneous source terms contain shell-dependent symmetry energy expressions (quadratic in isospin), and include, as well, an independently derived shell-model Coulomb energy equation which describes all known Coulomb displacement energies with a standard deviation of $\sigma_{\mathrm{c}}=41 \mathrm{keV}$. Perturbations of higher order in isospin, previously recognized as a cause of systematic effects in long-range mass extrapolations, are also incorporated. The most general solutions of the inhomogeneous difference equation have been deduced from a $\chi^{2}$-minimization procedure based on the recent atomic mass adjustment of Wapstra, Audi, and Hoekstra. Subjecting the solutions further to the condition of charge symmetry preserves the accuracy of Coulomb energies and allows mass predictions for nuclei with both $N \geqslant Z$ and $Z>N$. The solutions correspond to a mass equation with 470 parameters. Using this equation, 4385 mass values have been calculated for nuclei with $A \geqslant 16$ (except $N=Z=$ odd for $A<40$ ), with a standard deviation of $\sigma_{\mathrm{m}}=194 \mathrm{keV}$ from the experimental masses. 1988 Academic Press, Inc.
\end{abstract}

* Supported in part by the U.S. National Science Foundation and the U.S.-Israel Binational Science Foundation 


\section{CONTENTS}

INHOMOGENEOUS PARTIAL DIFFERENCE EQUATIONS AND GENERAL SOLUTIONS $\ldots \ldots \ldots \ldots \ldots \ldots \ldots \ldots, 274$

COULOMB ENERGIES, SYMMETRY ENERGIES, AND HIGHER-ORDER EFFECTS IN ISOSPIN ......... 275

METHOD OF MASS PREDICTION

\section{INHOMOGENEOUS PARTIAL DIFFERENCE EQUATIONS AND GENERAL SOLUTIONS}

Binding energy (or mass) relations can be written as sums and differences of the binding energies of a few neighboring nuclei (see, for example, Ref. 1) as

$$
\sum_{(i, j)} a_{i j}(N, Z) B(N+i, Z+j)=\tau(N, Z)
$$

Equation (1) can be applied recursively to predict new binding energies if only one value $B(N, Z)$ in this equation is unknown. The physical contents of Eq. (1) are contained in the structure of the relation, the coeffcients $a_{i j}(N, Z)$, and in the inhomogeneous source term $\tau(N, Z)$.

Relations are called homogeneous if the source term $\tau(N, Z) \equiv 0$; otherwise they are inhomogeneous. They are called hybrid relations if the coefficients $a_{i j}(N$, $Z)$ and/or the inhomogeneous source term $\tau(N, Z)$ are not constant but contain an explicit dependence on $(N$, $Z$ ). Such hybrid binding energy relations therefore have certain properties in common with binding energy equations.

The left-hand side of Eq. (1) can be expressed in terms of a partial difference operator $O^{m}$ of order $m$ if the coefficients $a_{i j}(N, Z)$ are independent of $N$ and $Z$. Properties of several second- to fourth-order difference equations

$$
O^{m} B(N, Z)=\tau(N, Z)
$$

have been discussed recently. ${ }^{2}$ Of particular interest is the third-order difference operator $O^{m}$ which is contained in the transverse Garvey-Kelson relation. ${ }^{3}$ This operator can be written as a product of three first-order operators,

$$
O^{m} B(N, Z)=\Delta^{1,-1} \Delta^{1,0} \Delta^{0,1} B(N, Z)
$$

with

$$
\Delta^{i, j} f(N, Z)=f(N, Z)-f(N-i, Z-j) .
$$

It represents ${ }^{4}$ the difference with respect to neutron excess of the effective neutron-proton interaction $I_{\mathrm{np}}(N$, $Z)=\Delta^{1,0} \Delta^{0,1} B(N, Z)$. Equation (3) can be approximately written as

$$
\begin{aligned}
O^{m} B(N, Z) & \approx\left(\frac{\partial}{\partial N}-\frac{\partial}{\partial Z}\right) I_{\mathrm{np}}(N, Z) \\
& \approx\left(\frac{\partial}{\partial N}-\frac{\partial}{\partial Z}\right) \frac{\partial^{2}}{\partial N \partial Z} B(N, Z) .
\end{aligned}
$$

The solutions of the inhomogeneous partial difference equation (2) represent binding energy expressions. They are of the form

$$
B(N, Z)=B_{\text {inhom }}(N, Z)+B_{\text {hom }}(N, Z),
$$

where $B_{\text {inhom }}(N, Z)$ represents a special solution of the inhomogeneous equation, and $B_{\text {hom }}(N, Z)$ represents the most general solution of the homogeneous equation. In particular, if $O^{m}$ is given by Eq. (3), the binding energy expression becomes

$$
\begin{aligned}
B(N, Z)=B_{\text {inhom }}(N, Z)+ & G_{1}(N) \\
& +G_{2}(Z)+G_{3}(N+Z)
\end{aligned}
$$

with point functions $G_{i}(k)$. This is a many-parameter binding energy (or mass) equation. Once the functions $G_{i}(k)$ are constructed from a $\chi^{2}$ minimization of the differences $B(N, Z)-B_{\text {exp }}(N, Z)$, Eq. (7) can be used to predict binding energies and masses of unknown nuclei. 


\section{COULOMB ENERGIES, SYMMETRY ENERGIES, AND HIGHER-ORDER EFFECTS IN ISOSPIN}

Coulomb and symmetry energies (taken for example in the analytical form of the liquid drop model) are expected to be responsible for small inhomogeneous source terms in Eq. (2) with $O^{m}$ from Eq. (3). Statistical tests, while compatible with this expectation, provide only weak evidence for the existence of inhomogeneous source terms (see, for example, Refs. 4 and 5).

Only recently ${ }^{1,4}$ has a new type of test established the need for inhomogeneous terms as well as their general characteristics. In this test all available mass data are systematically divided into subsets. The parameters are determined from one subset, and the remaining data are compared to the predictions.

An example is displayed in Fig. 1. Here, the residuals, that is, the differences between experimental and calculated masses, are shown. Only nuclei with a neutron excess $E=N-Z$ in the range $E_{\text {stab }}-\frac{1}{2} W<E$ $\leqslant E_{\text {stab }}+\frac{1}{2} W$ with $E_{\text {stab }}=0.4 A^{2} /(200+A)$ and $W=8$ were used to determine the point functions $G_{i}(k)$. The empty band along the stability line represents this subset of nuclei. Their mass values are reproduced very well with a standard deviation $\sigma_{\mathrm{m}}=145 \mathrm{keV}$. The figure displays only the residuals for the extrapolated neutronrich and proton-rich nuclei, which for this particular example display large systematic deviations (see below).

The influence of the inhomogeneous source term $\tau(N, Z)$ in Eq. (2) can very easily be established if $\tau(N$, $Z$ ) is written as

$$
\tau(N, Z)=O^{m} B_{\text {inhom }}(N, Z) .
$$

The function $B_{\text {inhom }}(N, Z)$ will then represent one special solution of the inhomogeneous partial difference equation (2).

The function $B_{\text {inhom }}(N, Z)$ was constructed with contributions from the Coulomb energy, $E_{\text {coul }}$, the nuclear isospin-dependent symmetry energy, $E_{\text {sym }}$, and terms higher than quadratic in isospin, $E_{\mathrm{sym}}^{\prime}$. It will be written as

$$
\begin{aligned}
B_{\text {inhom }}(N, Z)=-E_{\text {coul }} & (N, Z) \\
& -E_{\text {sym }}(A, T)-E_{\text {sym }}^{\prime}(A, T),
\end{aligned}
$$

where $T=\frac{1}{2}|(N-Z)|$.

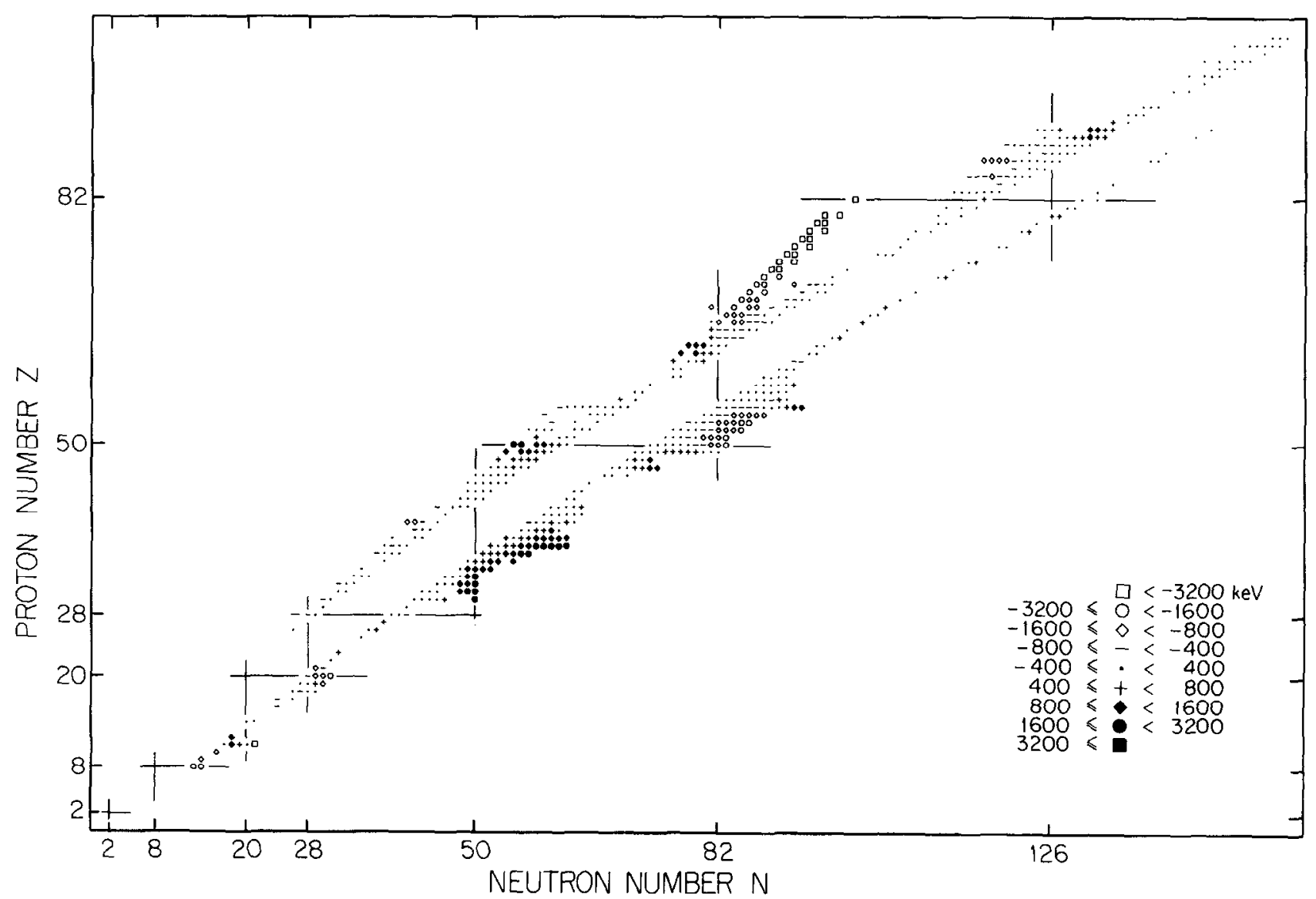

Figure 1. Differences between experimental masses and masses calculated using Eq. (7) for case II of Table B. The symbols in the figure represent nuclei with residuals in the indicated energy ranges. The figure displays only residuals for extrapolated mass values. 
Binding energies will contain separate contributions from nuclear energies and from Coulomb energies if isospin $T$ is a good quantum number. Therefore, Eq. (7) with $B_{\text {inhom }}(N, Z)$ from Eq. (9) must separate accordingly into nuclear and Coulomb terms,

$$
B(N, Z)=B_{\text {nucl }}(N, Z)+B_{\text {coul }}(N, Z),
$$

where $B_{\text {nucl }}(N, Z)$ satisfies charge independence of nuclear forces and hence charge symmetry. More explicitly, we have

$$
\begin{array}{r}
B_{\text {nucl }}(N, Z)=-E_{\text {sym }}(A, T)-E_{\text {sym }}^{\prime}(A, T)+G_{1}(N) \\
+G_{2}(Z)+G_{3}(A)
\end{array}
$$

and

$$
B_{\text {coul }}(N, Z)=-E_{\text {coul }}(N, Z),
$$

where the requirement of charge symmetry is achieved by imposing the constraint

$$
G_{1}(k)=G_{2}(k) \text {. }
$$

Similarly, the partial difference equation (2) with its third-order operator $O^{m}$ from Eq. (3) will separate into a Coulomb energy relation and a relation for nuclear energies which are both independently valid. ${ }^{6}$ The Coulomb energy relation describes Coulomb displacement energies between isobaric analog states, and it has a structure similar to the charge-symmetric KelsonGarvey relation $^{7}$ (see also Ref. 8). The relation for the nuclear energies is a relation for the excitation energies of isobaric analog states. ${ }^{6}$

The heuristic proof for the transverse GarveyKelson relation makes use of Hartree-Fock or Nilssonlike single-particle orbits (see Fig. 2 of Ref. 8). Separating the single-particle energies and the energies of interacting pairs into nuclear and Coulomb contributions on the basis of isospin $T$ as a good quantum number leads to the two independent relations. Coulomb energies and Coulomb interactions are accounted for separately, and the picture for the nuclear energies assumes identical fourfold-degenerate orbits for the protons and the neutrons. The relation obviously satisfies charge symmetry.

The Thomas-Ehrman shift in light proton-rich nuclei is a manifestation of the breakdown of the above picture signifying that isospin $T$ is not a good quantum number. For nuclei with unbound protons or weakly bound protons, corresponding proton and neutron orbits are not completely equivalent. The Thomas-Ehrman shift of up to several hundred $\mathrm{keV}$ in very-protonrich nuclei permits an estimate of the error which is made in these few extreme cases. It should be noted that separating the nuclear and Coulomb contributions by introducing charge symmetry of nuclear forces via the condition $G_{1}(k)=G_{2}(k)$ extends the applicability of Eq. (7) to all nuclei on both sides of the $N=Z$ line. Hence, unlike the transverse Garvey-Kelson relation and mass equation which are restricted to $N>Z$ and $N=Z$ $=$ even, Eq. (7) is capable of describing nuclei with $Z$ $>N$. It also has the benefit of a significant reduction in the number of free parameters.

A shell-model Coulomb energy equation ${ }^{9}$ has been used to represent $E_{\text {coul }}(N, Z)$. The parameters describing the Coulomb interactions and the nuclear size were derived from over 250 experimental Coulomb displacement energies in nuclei with $4<A<240$. These data are reproduced with a standard deviation of $\sigma_{\mathrm{c}}=41$ $\mathrm{keV}$. This value of the standard deviation is preserved in Eq. (7) because the remainder of the equation satisfies charge symmetry.

The nuclear symmetry energy depends quadratically on neutron excess. In a simple shell-model approach it is proportional to $T(T+1)$, whereas the Wigner super-multiplet model yields an expression with $T(T+4)$. The equation

$$
E_{\mathrm{sym}}(A, T)=\frac{a(A)}{A} T(T+1)
$$

with essentially constant but shell-dependent coefficients $a(A)$ was found ${ }^{4}$ to represent the nuclear symmetry energies rather well. However, in an extension of this approach it was observed that the terms linear and quadratic in isospin seem to follow a different dependence on nucleon number $A$. In the present work the symmetry energy was therefore written as

$$
E_{\mathrm{sym}}(A, T)=\frac{1}{A} \sum_{i=1}^{2}\left[a_{i}+b_{i}\left(A-A_{0}\right)\right] T^{i},
$$

allowing for a linear $A$ dependence in addition to the factor $1 / A$. The coefficients $a_{1}, a_{2}, b_{1}$, and $b_{2}$ were obtained (see Table $A$ ) from an iterative adjustment for each major shell region prior to the calculation of the point functions $G_{i}(k)$. Only data along the line of $\beta$ stability with $W=8$ were used as input to ensure a regular and uniform data base.

Using $E_{\text {coul }}(N, Z)$ and $E_{\text {sym }}(A, T)$ to initially represent the inhomogeneous contributions, the functions $G_{1}(N), G_{2}(Z)$, and $G_{3}(A)$ of Eq. (7) were calculated subject to the constraint $G_{1}(k)=G_{2}(k)$. Figure 1, previously mentioned, shows the results for the extrapolated neutron- and proton-rich nuclei. Again, only mass values for nuclei along the line of $\beta$ stability with $W=8$ were used as input. As observed earlier, ${ }^{4}$ the inclusion of inhomogeneous contributions from the Coulomb and symmetry energies yields only modest improvements in long-range extrapolations (compare Fig. 1 with Fig. 3 of Ref. 8).

Contributions of higher order in isospin, particularly contributions cubic in isospin, ${ }^{1,4}$ are strongly indicated by these results. These additional contributions 
TABLE A

Coefficients for the Symmetry Energies $E_{\text {sym }}(A, T)$ and $E_{\text {sym }}^{\prime}(A, T)$

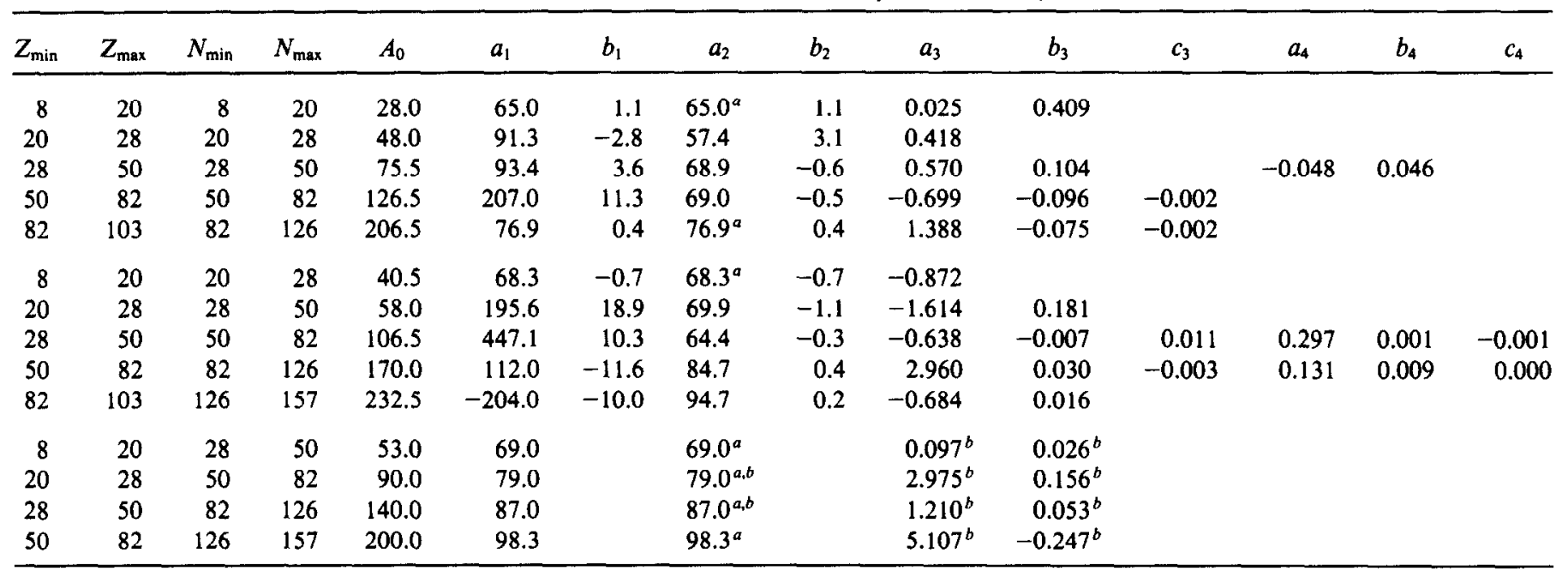

${ }^{a}$ A dependence on isospin of the form $T(T+1)$ was assumed.

${ }^{b}$ Estimated.

were therefore written for each major shell region as

$$
\begin{aligned}
E_{\mathrm{sym}}^{\prime}(A, T)=\frac{1}{A} \sum_{i=3}^{4}\left[a_{i}\right. & +b_{i}\left(A-A_{0}\right) \\
& \left.+c_{i}\left(A-A_{0}\right)^{2}\right]\left(T-T_{\mathrm{stab}}\right)^{i}
\end{aligned}
$$

with $T_{\text {stab }}=0.2 A^{2} /(200+A)$. Cubic isospin contributions with coefficients linear or quadratic in nucleon number $A$ (beyond the factor $1 / A$ ) were sufficient for most shell regions, but quartic isospin contributions had to be included in three major shell regions. Equation (16) implies a source term $\tau(N, Z)$ in Eq. (2) which for each shell region is essentially constant (or linear in isospin). The method of determining the coefficients of the cubic (and quartic) correction terms is described in the next section.

It is not unreasonable to expect higher-order effects in isospin, just as the description of rotational bands in (ordinary) spin space requires terms with [ $J(J$ $+1)]^{2}$ and beyond. In a shell-model picture the higherorder isospin terms are the result of departures from simple seniority or isospin coupling schemes, of subshell mixtures and of core excitations. Furthermore, the effect of deformed nuclear shapes on ground-state binding energies has the characteristics of an isospin dependence. This may also be responsible for the different $A$ dependences of the linear and quadratic isospin terms. Knowledge about these higher-order effects is minimal, and the approach taken in this work was phenomenological, but with the hope that some insight could be gained.

\section{METHOD OF MASS PREDICTION}

The present mass predictions make use of Eq. (7), which is the solution of an inhomogeneous partial difference equation. The special inhomogeneous solution of Eq. (9) includes Coulomb and symmetry energy terms and higher-order terms in isospin. The point functions $G_{i}(k)$ were obtained from a $\chi^{2}$-minimization procedure using the recent mass evaluation of Wapstra et al. ${ }^{10}$ as a boundary condition. A computer program which makes use of sparse matrix subroutines was used to solve the system of typically 400 linear equations in 400 unknowns subject to the constraint $G_{1}(k)=G_{2}(k)$. A realistic distribution of weight factors was achieved by quadratically adding $100 \mathrm{keV}$ to the experimental uncertainties, and all standard deviations were calculated accordingly. This added energy has the characteristics of an uncertainty of the theory, and one obtains a value for $\chi^{2}$ per degree of freedom on the order of unity.

As mentioned before, $E_{\text {coul }}(N, Z)$ was taken from a recent shell-model Coulomb energy equation. ${ }^{9}$ The standard deviation of $\sigma_{\mathrm{c}}=41 \mathrm{keV}$ for reproducing the experimental Coulomb displacement energies is preserved in the present predictions.

The coefficients $a_{i}, b_{i}$, and $c_{i}$ for the symmetry energy terms $E_{\text {sym }}(A, T)$ and $E_{\text {sym }}^{\prime}(A, T)$ of Eqs. (15) and (16) are shown in Table $A$. As discussed before, the coefficients for $E_{\text {sym }}(A, T)$ were obtained from an adjustment of Eq. (15) to the Coulomb-energy-corrected binding energies along the line of $\beta$ stability. The extraction of the coefficients for the cubic (and quartic) isospin terms in $E_{\text {sym }}^{\prime}(A, T)$ of Eq. (16) was thwarted for a long time. It was then discovered that the point functions 


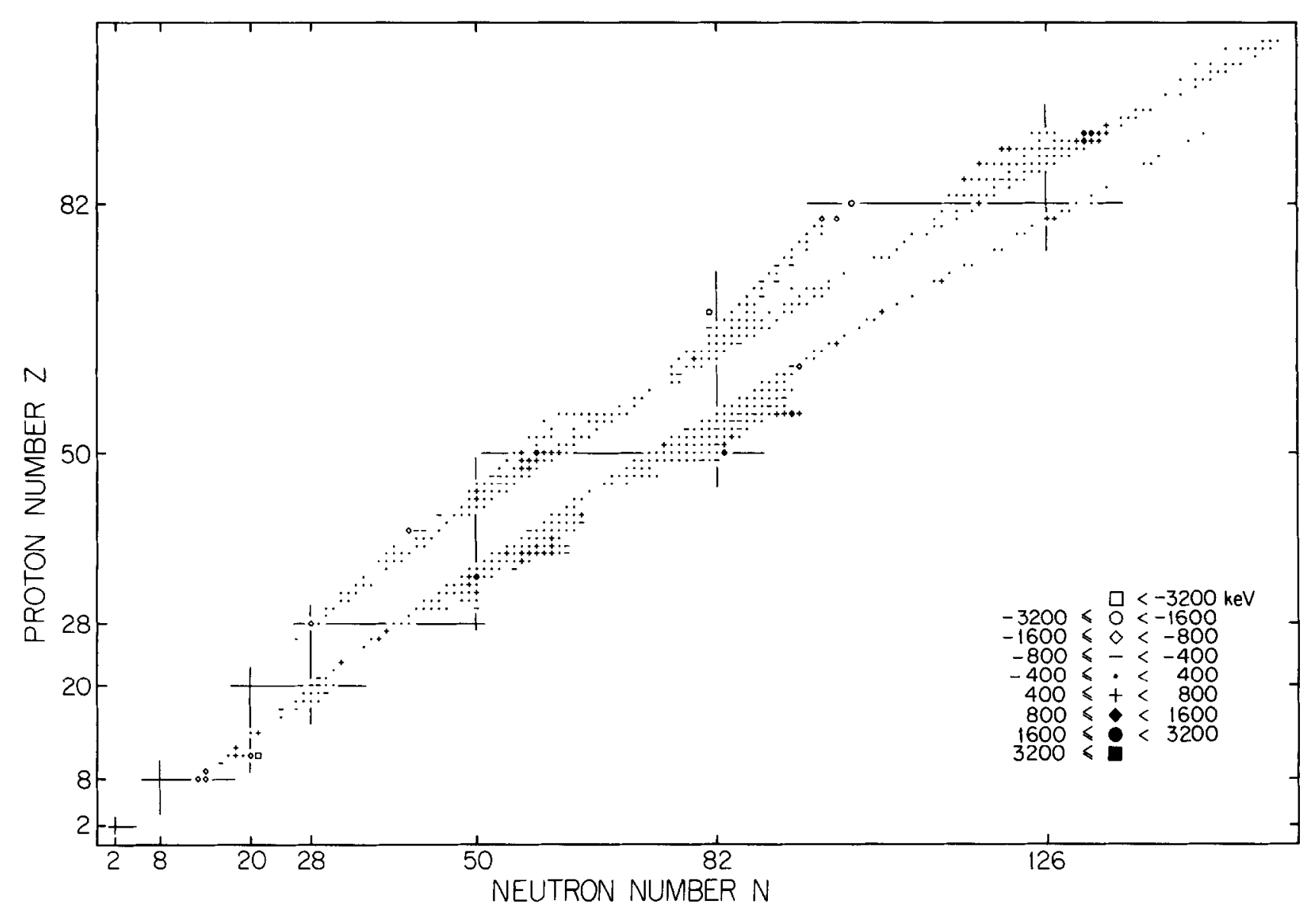

Figure 2. Differences between experimental masses and masses calculated using Eq. (7) for case III of Table B. The symbols in the figure represent nuclei with residuals in the indicated energy ranges. The figure displays only residuals for extrapolated mass values.

$G_{i}(k)$ implicitly generate cubic isospin contributions which have to be included together with the true effect displayed by the experimental data. The coefficients shown in Table A were obtained from an iterative adjustment to the residuals of the initial fit. Appropriate shifts were introduced in the shell regions not intersected by the stability line to guarantee the continuity of $\left(E_{\text {sym }}+E_{\text {sym }}^{\prime}\right)$ at shell crossings. In several shell regions with few or no data, cubic contributions were estimated to ensure that $\left(E_{\mathrm{sym}}+E_{\mathrm{sym}}^{\prime}\right)$ displays an acceptable change in slope at shell crossings. Extrapolations are very sensitive to the higher-order parameters, and a judicious limitation in the number of adjusted parameters was necessary.

Extrapolations obtained with the inclusion of cubic (and quartic) terms are displayed in Fig. 2. Com-

TABLE B

Mean Values and Standard Deviations of the Residuals Obtained with Different Inhomogeneous Terms and Data Bases for Fits to Nuclei with $N>Z$ and $N=Z=$ even

\begin{tabular}{rlllllllr}
\hline & & & & & & \multicolumn{2}{c}{ Mean and standard deviation (keV) } \\
\cline { 7 - 9 } Case & Data base & $E_{\text {coul }}$ & $E_{\text {sym }}$ & $E_{\text {sym }}^{\prime}$ & All nuclei & p-Rich & Stable & n-Rich \\
\hline I & Stable & No & No & No & $-41 \pm 695$ & $-445 \pm 1468^{a}$ & $0 \pm 79^{b}$ & $251 \pm 568^{a}$ \\
II & Stable & Yes & Yes & No & $-36 \pm 564$ & $-260 \pm 1081^{a}$ & $0 \pm 145$ & $71 \pm 710^{a}$ \\
III & Stable & Yes & Yes & Yes & $-3 \pm 231$ & $-12 \pm 347$ & $0 \pm 145$ & $-4 \pm 336^{a}$ \\
IV & All & Yes & Yes & Yes & $0 \pm 188$ & $6 \pm 194$ & $-1 \pm 169$ & $-4 \pm 246$ \\
\hline
\end{tabular}

Note. The "stable" subset of nuclei is defined by $E_{\text {stab }}-\frac{1}{2} W<E \leqslant E_{\text {stab }}+\frac{1}{2} W$ with $E=N-Z, E_{\text {stab }}=0.4 A^{2} /(200+A)$, and $W=8$. The inhomogeneous terms $E_{\text {coul }}, E_{\text {sym }}$, and $E_{\text {sym }}^{\prime}$ are included as indicated. The point functions $G_{1}(N)$ and $G_{2}(Z)$ are subject to the constraint $G_{1}(k)=G_{2}(k)$.

${ }^{a}$ Extrapolated.

${ }^{b}$ Functions $G_{1}(N)$ and $G_{2}(Z)$ are unconstrained. 
TABLE C

Statistical Data for the $\chi^{2}$ Minimization

\begin{tabular}{lrrr}
\hline & \multicolumn{2}{c}{ Region } & \\
\cline { 2 - 3 } & \multicolumn{1}{c}{$R$} & $S$ & $\begin{array}{c}\text { All } \\
\text { nuclei }\end{array}$ \\
\hline$Z$ (min) & 8 & 9 & 8 \\
$Z(\max )$ & 103 & 30 & 103 \\
$N(\min )$ & 9 & 8 & 8 \\
$N(\max )$ & 157 & 29 & 157 \\
Number of input data points & 1515 & 70 & 1585 \\
Numbe of parameters & 470 & 0 & 470 \\
Number of predicted mass values & 4054 & 331 & 4385 \\
Standard deviation $\sigma_{\mathrm{m}}(\mathrm{keV})$ & 188 & 288 & 194 \\
Standard deviation $\sigma_{\mathrm{c}}(\mathrm{keV})$ for & 41 & & \\
$\quad$ Coulomb displacement energies & & & \\
\hline
\end{tabular}

Note. Region $R$ contains nuclei with $N>Z$ and $N=Z=$ even. Region $S$ contains nuclei with $N<Z$.

parison with Fig. 1 clearly shows the great improvement in the extrapolations. This information is quantified in Table B, which shows mean values of the residuals and the associated standard deviations for all nuclei and for the subsets of p-rich, stable, and n-rich nuclei. The large mean values for the extrapolated regions of p-rich and $\mathrm{n}$-rich nuclei become small only when the higher-order terms in isospin, $E_{\text {sym }}^{\prime}(A, T)$ are included (compare cases II and III). The first three entries of Table B, cases I, II, and III, correspond to Fig. 3 of Ref. 8 and Figs. 1 and 2 of this contribution, respectively.

The entry in the last row of Table B, case IV, shows means and standard deviations for the case where all known nuclidic masses are included in the data base for determining the point functions $G_{i}(k)$. Interestingly, the various standard deviations are quite stable and change only moderately from case III to case IV. The standard deviations for the p-rich and n-rich nuclei improve at some expense to the stable nuclei. The overall standard deviation improves from 231 to $188 \mathrm{keV}$. The statistical data for this $\chi^{2}$ minimization are given in Table $\mathrm{C}$. The standard deviation for reproducing the input data of approximately 1500 mass values is 188 $\mathrm{keV}$. The total number of parameters for the functions $G_{i}(k)$ and the coefficients of $E_{\text {sym }}(A, T)$ and $E_{\text {sym }}^{\prime}(A, T)$ is $391+79=470$.

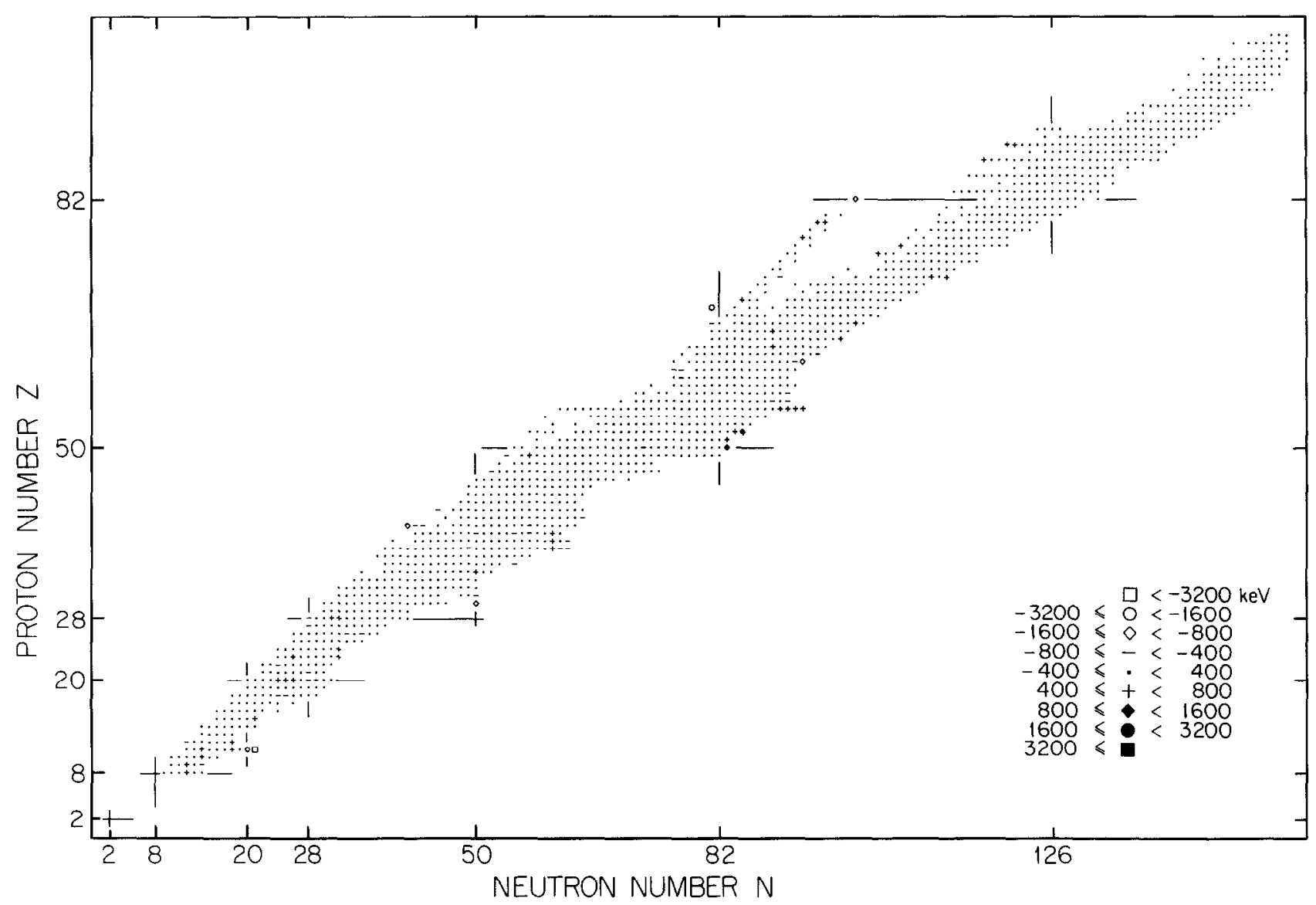

Figure 3. Differences between experimental masses and masses calculated using Eq. (7) for case IV of Table B. The symbols in the figure represent nuclei with residuals in the indicated energy ranges. All available data were used to determine the mass parameters; the figure displays the entire data base of region $R$. 
Nuclei with $Z>N$ were not included in determining any of the parameters. The uncertainties for reproducing their masses is a reflection of the uncertainties for predicting the masses of the corresponding light neutron-rich nuclei combined with the uncertainties for predicting Coulomb energies. ${ }^{9}$ The statistical information for this group of nuclei is included in Table $\mathrm{C}$.

Individual residuals for the global fit of region $R$ are displayed in Fig. 3. One can see that a few very-neutron-rich and very-proton-rich nuclei such as ${ }^{31,32} \mathrm{Na}$ and ${ }^{149} \mathrm{Er}$ are poorly reproduced. This is in part due to the constraint $G_{1}(k)=G_{2}(k)$ but also reflects the strong sensitivity of extrapolations to the characteristic behavior of the higher-order isospin terms. Long-range extrapolations with the present approach must therefore be considered with caution; their quality may well be shell dependent. Improvements may become possible in the future based on a better understanding of the origin of the perturbations of higher order in isospin.

\section{References}

1. J. Jänecke, in Proceedings, 7th International Conference on Atomic Masses and Fundamental Constants (AMCO-7), edited by O. Klepper (Darmstadt 1984), p. 420
2. J. Jänecke and P. J. Masson, Phys. Rev. C 32, 1390 (1985)

3. G. T. Garvey and I. Kelson, Phys. Rev. Lett. 16, 197 (1966)

4. J. Jänecke and E. Comay, Nucl. Phys. A 436, 108 (1985)

5. J. Jänecke and H. Behrens, Phys. Rev. C 9, 1276 (1976)

6. J. Jänecke, in Isospin in Nuclear Physics, edited by D. H. Wilkinson (North-Holland, Amsterdam, 1969), Chap. 8, p. 298

7. I. Kelson and G. T. Garvey, Phys. Lett. 23, 689 (1966)

8. J. Jänecke and P. J. Masson, ATOMIC DATA AND NUClEAR DATA TABles 39, 265 (1988)

9. E. Comay and J. Jänecke, Nucl. Phys. A 410, 103 (1983); J. Jänecke and E. Comay, Phys. Lett. B 140, 1 (1984)

10. A. H. Wapstra, G. Audi, and R. Hoekstra, ATomiC DATA AND Nuclear DATA TABles 39, 281 (1988) 